\title{
Flexible DLC film coated rubber: friction and the effect of viscoelastic deformation of rubber substrate
}

\author{
Y. T. Pei, D. Martinez-Martinez \& J. Th. M. De Hosson \\ Materials innovation institute M2i, Department of Applied Physics, \\ Zernike Institute for Advanced Materials, University of Groningen, \\ The Netherlands
}

\begin{abstract}
This paper focuses on the frictional behavior of flexible diamond-like carbon (DLC) films coated hydrogenated nitrile butadiene rubber. By making use of the substantial thermal mismatch between DLC film and rubber substrate, the DLC film splits during deposition into micro-segments that contribute to flexibility. The size of film micro-segments can be tuned by controlling the temperature variation of rubber substrate during deposition. The influence of the size of DLC film micro-segments on the frictional performance is studied. The effect of viscoelasticity of the rubber substrate on the frictional behavior of DLC film coated system is scrutinized with tribo-tests and theoretical analysis. The importance of adhesive and hysteresis contributions to friction is revealed, and an overarching model is presented.
\end{abstract}

Keywords: DLC film, flexibility, viscoelastic deformation, rubber substrate, tribology.

\section{Introduction}

The coefficient of friction ( $\mathrm{CoF}$ ) of rubbers sliding against rigid solids is associated with contributions from interfacial adhesion and from energy losses in the bulk of the rubbers. Both terms are directly related to the viscoelastic properties of a rubber [1]. By applying a chemically inert coating such as diamond-like carbon (DLC) film on rubber substrates, the interfacial adhesion between the sliding counterparts may be largely restrained [2, 3]. On the other hand, a thin film may only reduce partially the inevitable viscoelastic 
deformation of coated rubbers and thus the associated energy dissipation via internal damping, which are affected by the loading/sliding conditions.

In the past a tile-like patterned films on rubber was proposed and deposited by using a net mask in front of the substrate [4]. Obviously, there are technical problems with this approach: the size of film segments is rather limited to submillimeter and especially the open gap that produced a large amount of debris and led to a high friction and severe wear of coated rubber [3]. Another approach was recently developed for depositing micro-segmented DLC films of superior flexibility on rubber [5]. The novelty is that the segmentation process of DLC films is self-adjusting throughout the deposition and the size of film segments separated by crack network can be well controlled at much smaller length scales. In this paper, the frictional behavior of DLC film coated HNBR rubber is elucidated with tribo-tests and theoretical modeling as a whole system based on the soft contact and visco-elasticity of the rubber substrate.

\section{Experimental}

Hydrogenated nitrile butadiene rubber (HNBR) sheets of $2 \mathrm{~mm}$ thickness were used as substrate. The wax removal and cleaning processes of HNBR substrates $\left(45 \times 45 \mathrm{~mm}^{2}\right.$ size $)$ have been described in detail elsewhere [5]. Plasma cleaning treatment of rubber substrates and successive plasma assisted CVD (PACVD) deposition of DLC films were carried out in a Teer UDP400/4 closed-field unbalanced magnetron sputtering system with all the magnetrons powered off. A pulsed DC power unit (Pinnacle plus, Advanced Energy) was used for substrate bias source, operating at $250 \mathrm{kHz}$ and $87.5 \%$ duty cycle for plasma cleaning and deposition of DLC films. The ratio of gas flow rates was set at $\mathrm{Ar}_{2} \mathrm{C}_{2} \mathrm{H}_{2}=3: 2$, and constant process pressure of $3 \times 10^{-3}$ mbar. The detailed PACVD process is referred to our previous work [5]. The DLC films are named as DLCxxx-yyyV, e.g. DLC600-400V, where $\mathrm{xxx}$ is the substrate bias voltage in volt used for plasma cleaning and yyy the bias voltage for successive PACVD deposition.

The coated rubber sheets were glued onto ø30 mm polished M2 steel discs for tribotests. The tests were performed at room temperature $\left(20-23^{\circ} \mathrm{C}\right)$ on a CSM tribometer with ball-on-disc configuration. The variation of depth of the wear track was in-situ monitored with a resolution of $0.02 \mu \mathrm{m}$ by a rotary variable differential transformer sensor during the tribo-tests. The counterpart was commercial ø6 mm 100Cr6 steel balls of hardness HRC 60-62. All the tribotests were carried out at a sliding velocity of $10 \mathrm{~cm} \mathrm{~s}^{-1}$ and a constant humidity of $35 \pm 1 \%$ kept with a humidity regulator. The wear track of DLC film coated rubber samples were investigated with scanning electron microscope (SEM) (Philips XL-30 FEG). 


\section{Results}

\subsection{Frictional behavior of DLC film coated HNBR rubber}

The density of crack network or film segment size affects the flexibility and consequently the friction of DLC films coated HNBR rubber. Fig. 1 shows the tribological result of the DLC films of different segment sizes and also the CoF curve of uncoated HNBR as the reference that is ten times larger. All the CoF curves of DLC films start at an initially low value of $0.11 \sim 0.12$ at the beginning of sliding and gradually increase to a steady state of higher values. Clearly, the DLC films exhibit a direct relation between the average size of film segments and the steady-state CoF: the smaller the film segments are, the lower the steadystate $\mathrm{CoF}$ is and also the shorter time to reach it. The initial low CoF reflects the "real" friction of DLC films against the bearing steel ball. The significant increase of $\mathrm{CoF}$ in the onset of tribo-tests is attributed to the visco-elastic deformation of rubber substrates.
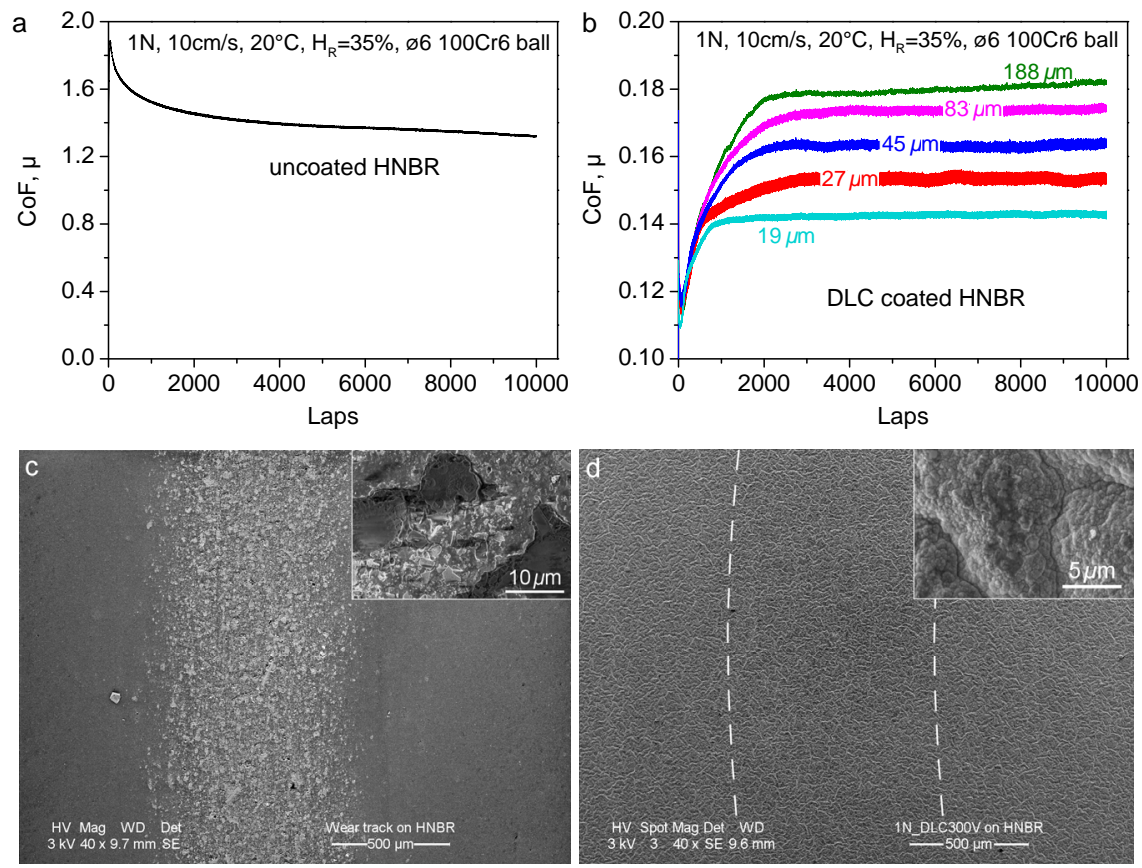

Figure 1: Coefficient of friction of uncoated HNBR (a) and $300 \mathrm{~nm}$ thick DLC films coated HNBR rubber with film segment sizes indicated (b). SEM micrographs showing the wear track of (c) uncoated

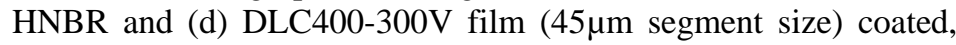
with the inset highlights the wear characters. 
The wear tracks of DLC film coated HNBR rubber are hardly visible in an overview of low magnification (see Fig. 1(d)), indicating superb protection to rubber substrates. It is in sharp contrast to the heavily damaged wear track of uncoated HNBR shown in Fig. 1(c), where a layer distinguishable from the bulk rubber was formed on the wear track. This layer consisting of debris and partly carbonized rubber scale was called "dead layer" with modified properties [6] and formed due to high flash temperatures during sliding and adhesive interactions between rubber and counterpart.
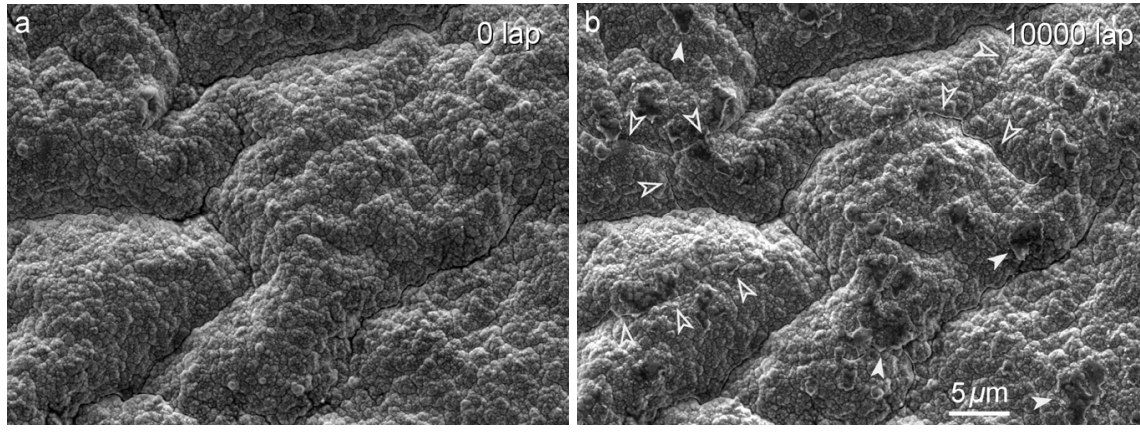

Figure 2: $\quad$ SEM micrographs showing the wear morphology of the same area in a wear track on DLC400-300V film (45 $\mu \mathrm{m}$ segment size) tested at $3 \mathrm{~N}$ load: (a) as deposited and (b) after sliding 10000 laps. Open arrows indicate new fracture cracks and close arrows point to the polished spots.
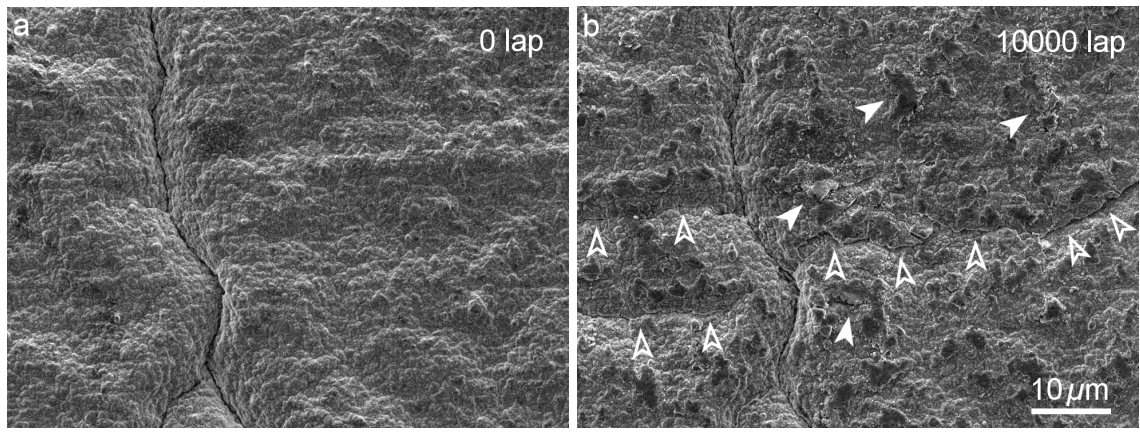

Figure 3: $\quad$ SEM micrographs showing the wear morphology of the same area in a wear track on the DLC400-400V film (188 $\mu \mathrm{m}$ segment size) tested at $3 \mathrm{~N}$ load: (a) as deposited and (b) after sliding 10000 laps. Open arrows indicate new fracture cracks and close arrows point to the debris.

SEM observations of the wear track in Fig. 2 revealed that DLC400-300V film of $45 \mu \mathrm{m}$ average segment size showed a few new fracture cracks of micrometers size formed in the first 1000 laps of sliding, and thereafter only tiny 
polished spots occurred on the humps of convex segments till sliding for 10000 revolutions. The majority of the surface area of the DLC film was intact after the tribo-test. In contrast, DLC films with large segment sizes fractured more heavily during sliding against steel ball. In DLC400-400V film of $188 \mu \mathrm{m}$ average segment size as shown in Fig. 3, newly formed long fracture cracks propagated through the entire segments in a brittle manner and caused the formation of debris that scratched both the counterpart and DLC film itself, leading to higher friction as shown in Fig. 1(b).

\subsection{Effect of the viscoelastic deformation of rubber substrate on friction}

In soft contact such as sliding against DLC film coated rubbers that deform viscoelastically, the tribological behavior may be rather different from that of hard contact sliding. Fig. 4(a) shows the effect of normal load on the CoF of DLC films coated HNBR rubber such that the steady-state CoF increases with increasing the normal load. All the CoF curves of a DLC film coated sample are very similar in shape but rise with increasing normal load. In particular, all the
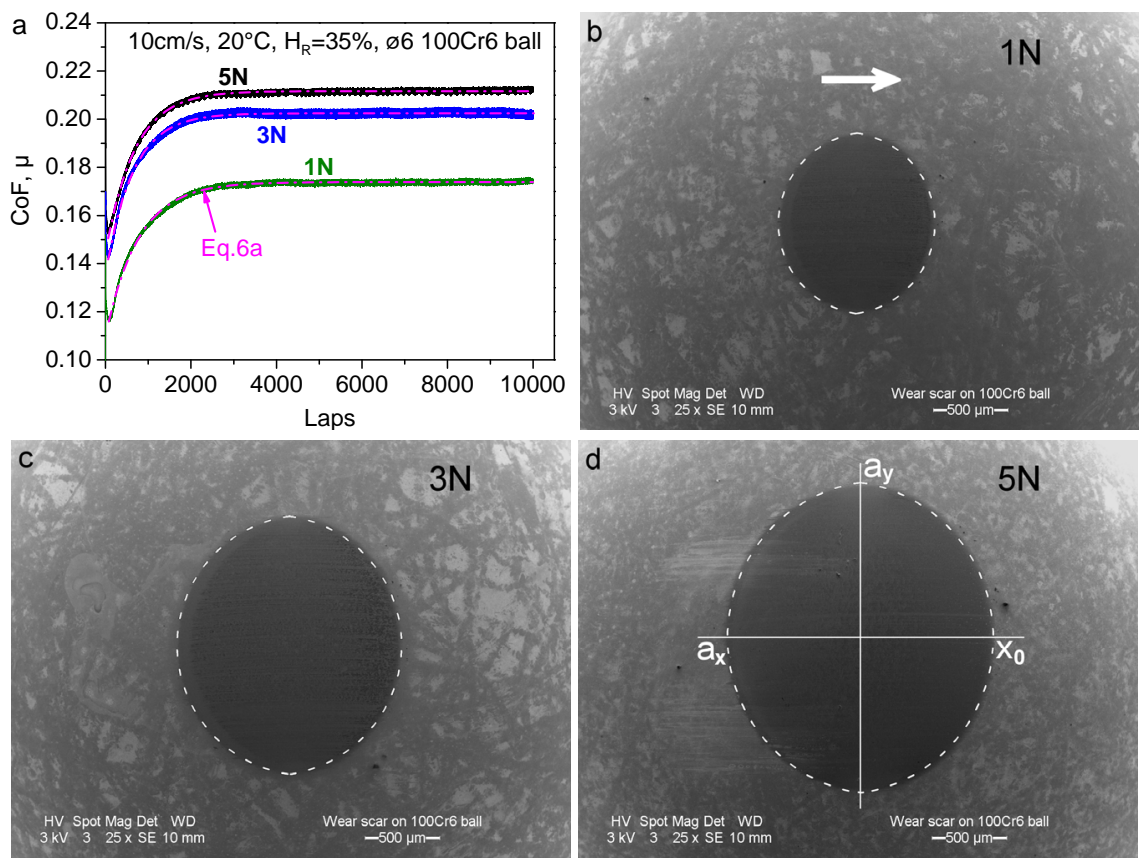

Figure 4: The coefficient of friction (a) and wear scar of ø6 mm 100Cr6 ball counterpart sliding against DLC400-300V film at normal load: (b) $1 \mathrm{~N}$, (c) $3 \mathrm{~N}$ and (d) $5 \mathrm{~N}$. An arrow indicates the sliding direction of the DLC film coated rubber disc. The contact areas predicted by the analytical model are included as dashed lines. The labels representing the dimensions of the contact area are depicted in (d). 
CoF curves exhibit a gradual increase of $\mathrm{CoF}$ at the onset of tribo-tests before reaching steady-state values. It is noteworthy that, for a given film, the variation of $\mathrm{CoF}(\Delta \mathrm{CoF})$ in a test is independent of the normal load. Both the increase of the steady-state CoF with normal load and the gradual increase of CoF at the onset of individual tests are related to the enlarged contact area due to the viscoelastic deformation of rubber substrate under loading. Fig. 4(b)-4(d) shows the wear scar of the ball counterpart after sliding 10000 laps at different normal loads, which provides a direct measure of the contact area. The wear scars are elliptical and symmetrical from the front side to the back side. At $1 \mathrm{~N}, 3 \mathrm{~N}$ and $5 \mathrm{~N}$ normal load, the contact area measured on the scars is $0.956 \mathrm{~mm}^{2}$, $1.992 \mathrm{~mm}^{2}$ and $2.812 \mathrm{~mm}^{2}$, respectively. These values are consistent with the calculated sizes of contact area according to Hertzian theory, being $0.959 \mathrm{~mm}^{2}$, $1.996 \mathrm{~mm}^{2}$ and $2.805 \mathrm{~mm}^{2}$, although the later assumes a circular contact area.

Fig. 5 shows the evolution of the measured depth of a wear track and the corresponding CoF curve of DLC400-300V film coated HNBR, which are very alike in trend. Before reaching steady-state, the viscoelastic deformation of the rubber substrate at one point during one pass is not completely recovered before the subsequent pass in the ball-on-disc configuration, and therefore the overall depth of the wear track increases and so do the contact area and CoF. The steady state is reached when the deformation at a point on the wear track is dynamically recovered in the interval between a preceding and subsequent lap, leading to a constant CoF due to little change in the contact area.

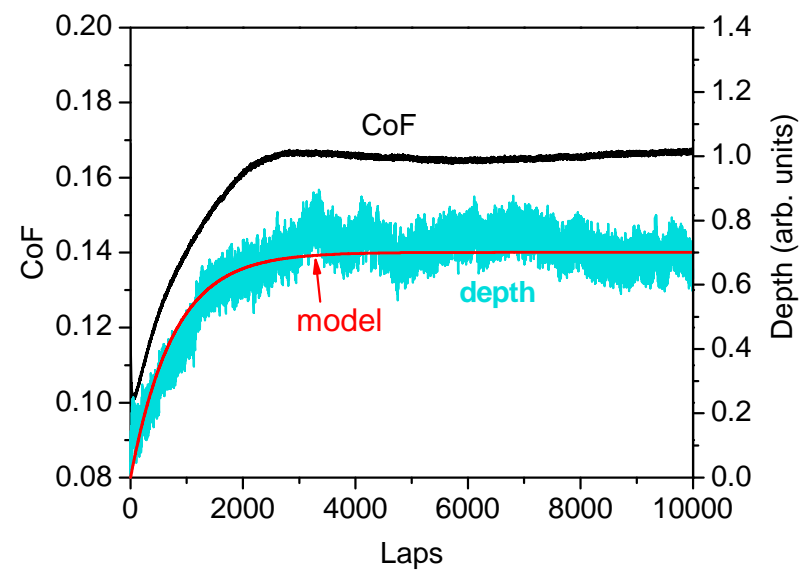

Figure 5: Evolution of $\mathrm{CoF}$ and wear track depth of DLC400-300V film coated HNBR versus sliding laps (revolutions).

\section{Discussion}

For solids subjected to elastic contact, the CoF was found to decrease with increasing the normal load [7]. This was interpreted by an adhesion mechanism associated with the change of contact area and expressed as: 


$$
\mu_{\text {adh }}=S \frac{A}{L}
$$

where $S$ is the shear strength between the surfaces in contact, $A$ is the contact area and $L$ is the load. In elastic (Hertzian) condition, the contact area depends on the load as $A \propto L^{n}$, with $\mathrm{n}=2 / 3$ for a circular contact (sphere on plane). Thus Eq. (1) can be re-written as:

$$
\mu_{a d h}=K_{a d h} L^{x}, \quad-1<x<0
$$

In our case, the DLC films are deposited on rubber substrates, and their viscoelastic influence has to be considered as well. The hysteresis contribution to friction $\left(\mu_{\text {hyst }}\right)$ is a consequence of the delayed response of the rubber to loading due to its viscoelasticity. It causes that a fraction of energy in deforming the rubber by the counterpart is lost, since the rubber recovery is not immediate. This component of friction can be expressed in a similar way as the adhesive contribution (Eq. (2)):

$$
\mu_{\text {hyst }}=K_{\text {hyst }} L^{y}, \quad 0<y<1
$$

where the value of the exponent $y$ depends on the specific model adopted. For instance, $y=1 / 3$ when using a modified elastic model, and $y=1 / 2$ for a 'mattress' approach composed of Voigt units [8]. Nevertheless, it is worth mentioning that the adhesive and hysteresis contributions show an opposite behavior with increasing load, which permits decoupling their relative influence. Therefore, the total CoF can be expressed as a combination of Eqs. (2) and (3) with $x=1 / 3$ :

$$
\mathrm{CoF}=\mu_{\text {adh }}+\mu_{\text {hyst }}=K_{\text {adh }} L^{-1 / 3}+K_{\text {hyst }} L^{y}, \quad 0<y<1
$$

Fitting of the initial and steady CoFs for sample DLC400-300V to Eq. (4) is depicted in Fig. 6(a) and 6(b), respectively. Only the fitting with $y=1 / 3$ is shown (i.e. using the modified elastic model). From this type of fitting the values of $K_{a d h}$ and $K_{\text {hyst }}$ can be estimated, and the comparison of the ratio $K_{\text {adh }} / K_{\text {hyst }}$ at the initial

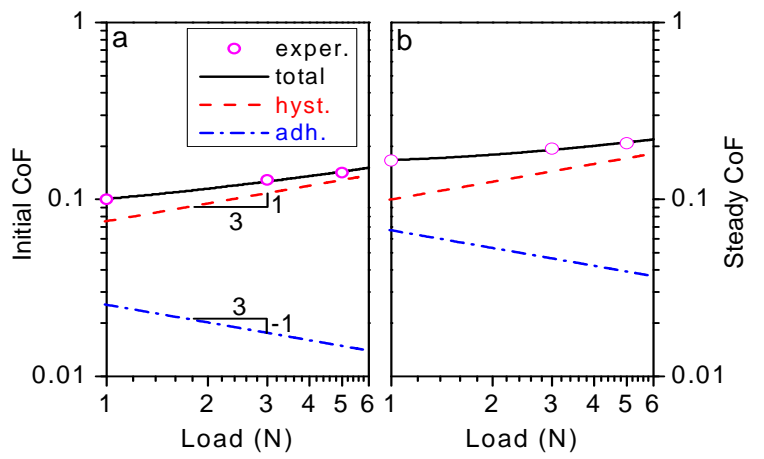

Figure 6: Decoupling of the adhesive and hysteresis contributions to friction versus normal load. Top: fitting of the initial (a) and steady $\mathrm{CoF}$ (b) of the sample DLC400-300V to Eq. 4 with $y=1 / 3$. 
and the steady states points at the contribution responsible for the increase in the total friction. It can be seen that the ratio $K_{a d h} / K_{\text {hyst }}$ grows from the initial to the steady states in all the cases, which indicates that the adhesive interaction is the dominant one responsible for the CoF increase during a tribotest.

According to Eq. (1), however, only an increase of the contact area $(A)$ between the counterpart ball and the DLC film sample may account for the observed CoF growth during a tribotest, since the shear strength and the load are constant for a given sample and tribotest, respectively. In order to verify this issue, an analytical model has been developed in order to simulate the viscoelastic behavior of rubber during cyclic tribotests [9]. This model reveals the evolution of both frictional components in tribotests carried out at different conditions. The adhesive one is derived through the calculus of contact area, while the hysteresis one is calculated by integration of forces and torques within the contact area.

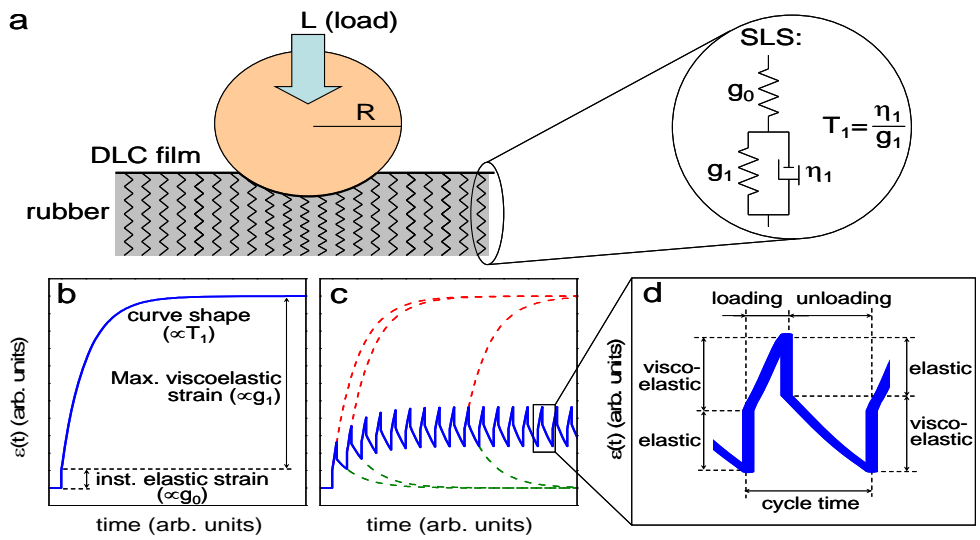

Figure 7: (a) Sketch of the mattress model composed of standard linear solid (SLS) units under deformation of a ball. The detailed SLS arrangement is enclosed. (b) Strain evolution of a SLS under a static loading. (c) Strain evolution of a SLS under cyclic loading. (d) A closer view of a loading-unloading cycle in steady state.

As depicted in Fig. 7(a), this model assumes that rubber can be approximated as a 'mattress' composed of individual elements without lateral interaction. As a result, this model is non-Hertzian, which leads to absence of deformations out of the contact area, and causes that the contact area and depth cannot be determined precisely at the same time. Each element of the mattress is a simple arrangement of two springs and a dashpot known as 'standard linear solid' (SLS), which is also sketched in Fig. 7(a). This structure is composed of one isolated spring that accounts for the instant elastic deformation of the rubber depending on the applied stress $\left(s_{0}\right)$ and the spring strength $\left(g_{0}\right)$. The spring is situated in series with a secondary array called 'Voigt unit' that represents the rubber viscoelasticity. Essentially, the Voigt unit can be understood as a 'delayed' 
spring. Its maximum deformation depends on the stress applied $\left(s_{0}\right)$ and the strength of the spring $\left(g_{1}\right)$, although the time needed to reach that deformation depends on the viscoelasticity of the dashpot $\left(\eta_{1}\right)$. Thus, the creep response function of the SLS $(\varepsilon(t))$, which represents the strain response to a unit change in stress, can be represented as [10]:

$$
\varepsilon(t)=s_{0}\left[\frac{1}{g_{0}}+\frac{1}{g_{1}}\left(1-\exp \left(-\frac{t}{T_{1}}\right)\right)\right], \text { with } T_{1}=\frac{\eta_{1}}{g_{1}}
$$

Fig. 7(b) represents this function, where the role of each element can be distinguished. In the case of a cyclic test, loading and relaxation are in succession, as depicted in Fig. 7(c). In each of them both elastic and viscoelastic deformations are present (see Fig. 7(d)). However, they never reach the maximum deformation nor the total relaxation (see dashed lines in Fig. 7(c)). As a consequence, the steady state is not achieved when the maximum deformation of the Voigt unit is reached, as occurred in the case of a quasi-static indentation (see Fig. 7(b)). In contrast, this condition is achieved when the deformation by one pass of loading equals the relaxation in the rest of the cycle (see Fig. 7(d)). Nevertheless, the overall trend keeps exponential, although its final shape depends on the influence of all SLS parameters $\left(g_{0}, g_{1}, \eta_{1}\right)$ and tribotest conditions (load, sliding velocity, track radius and contact frequency, ball diameter, etc.).

The SLS is the minimum arrangement that can reproduce the overall deformation behavior of the rubber substrate in pin-on-disc tribotests, although qualitatively. A real elastomer is more complex, and many Voigt units are needed to account for its behavior. Nevertheless, only a few units are active under given test conditions of sliding frequency and speed [11]. Therefore, the SLS has been parameterized first referring to real test conditions (e.g. $1 \mathrm{~N}$ load and $9 \mathrm{~mm}$ radius of wear track), in order to reproduce some experimental results such as the size and shape of the contact area at the end of a test. Both of them reveal that the rubber behaves as a quasi-elastic material (see Fig. 4). First, the size of contact area and the normal load can be correlated by Hertzian equation as indicated before. Second, the contact area is symmetric in the $\mathrm{x}$ axis, i.e. the semi-contact lengths of the contact area in the backward $\left(\mathrm{x}_{0}\right)$ and frontward $\left(\mathrm{a}_{\mathrm{x}}\right)$ are very similar $\left(x_{0} / a_{x} \sim 1\right)$. In addition, the elongation of the contact area is moderate $\left(a_{y} / a_{x} \sim 1.2\right)$. This observation also explains why the $\mathrm{CoF}$ reaches a steady state, since variations due to rubber viscoelasticity are only possible while the symmetry of contact area (i.e. $x_{0} / a_{x}=1$ ) is not reached [ix].

The other experimental result to be reproduced in the next step is the shape of the CoF curves (see Fig. 4(a)). After rubber parameterization to the aforementioned test conditions, simulations on other conditions are performed. To compare the experimental and simulated results, an exponential function has been selected for data fitting, since it reflects the viscoelastic influence of the rubber behavior (cf. Eq. (5)):

$$
C o F=C_{\text {steady }}-\Delta C o F \exp \left(-\frac{\text { laps }}{K}\right)
$$


where $\mathrm{CoF}_{\text {steady }}$ represents the steady state $\mathrm{CoF}, \triangle \mathrm{CoF}$ is the variation of $\mathrm{CoF}$ in a test and $K$ is a constant that represents the curvature of the curve. It is worth mentioning that an equivalent expression can be found when replacing the $\mathrm{CoF}$ with the contact area $(A)$ or wear track depth according to Eq. (1):

$$
A=A_{\text {steady }}-\Delta A \exp \left(-\frac{\text { laps }}{K}\right)
$$

The results of modelling and experiments are in good agreement as compared in Fig. 4(a) and Fig. 5. As being shown in Fig. 4(a), $\triangle C O F$ remains almost constant for a given sample, and the steady $\mathrm{CoF}$ grows with the load, as mentioned previously. The viscoelastic influence of rubber substrates can be appreciated not only on the frictional behavior, but also in the evolution of wear track depth during a tribotest (see Fig. 5). Its behavior follows the same trend as the CoF and reaches a steady state of viscoelastic deformation at a certain revolution of sliding. Since the underlying viscoelastic mechanism is the same, the shape of this curve is well reproduced by the model as seen in Fig. 5.

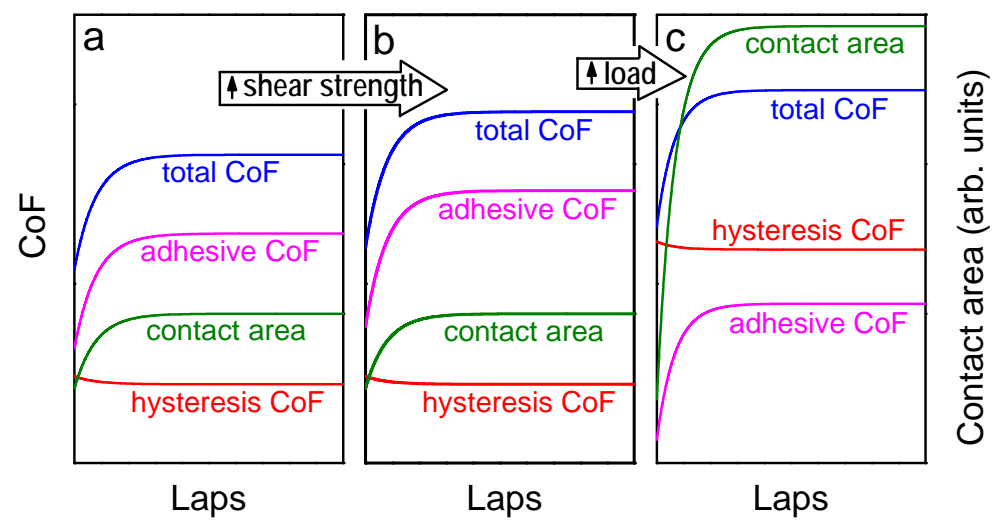

Figure 8: Schematic overview of the influence of the increase in shear strength (from (a) to (b)) and normal load (from b to c) on the contact area and friction contributions of DLC film coated rubber.

The overall behavior of DLC-coated HNBR can be summarized as follows (see Fig. 8). When a test is carried out at a constant load (Fig. 8(a)), the adhesive contribution grows during the test due to the increase of contact area caused by the viscoelastic deformation of rubber. Therewith, the hysteresis contribution is reduced due to the elongation of contact area [ix]. For two different DLC films deposited on the same type of rubber and tested in same conditions (Fig. 8(a) and 8(b)), the differences in $\mathrm{CoF}$ are mainly attributed to the different shear strength of the DLC films against the counterpart, since the viscoelastic response of the rubber maintains the same. When the load is increased (Fig. 8(b) and 8(c)), in contrast, the hysteresis component exhibits a major growth that is responsible for 
the increase of the total $\mathrm{CoF}$. Although the contact area also grows, the adhesive component of $\mathrm{CoF}$ decreases according to Eq. (2).

\section{Conclusion}

In summary, flexible DLC films of micrometer-scale segment minimize the friction of HNBR rubber. The denser crack network contributes to better flexibility and the ultra-low friction of DLC film coated HNBR rubber. The influence of adhesive and hysteric contributions to friction has been examined by means of experimental and theoretical analysis. As a result, an overarching model about their relative influence is presented. The frictional behavior of DLC film coated HNBR has been successfully correlated with the evolution of the contact area shape and size due to rubber viscoelasticity. In general, friction increases upon increasing load due to the growth of rubber hysteresis contribution. In contrast, the friction during a tribotest grows due to an increased adhesive contribution related to a larger contact area that is determined by the visocelasticity of rubber substrate. It can be concluded that the analyses presented provides generic design rules for the deposition of flexible and low friction films on dynamic rubber seals and an approach to drastically reduce energy consumption in bearings and lubrication systems.

\section{Acknowledgements}

This research was carried out under the project number MC7.06247 in the framework of the Research Programme of the Materials innovation institute M2 $\mathrm{i}$ (www.m2i.nl), Delft, the Netherlands. Financial support from the M2i is gratefully acknowledged. Dr. X.B. Zhou of the SKF Engineering and Research Centre in Nieuwegein, The Netherlands is thanked for his valuable discussion and input.

\section{References}

[1] K.A. Grosch, P. Roy. Soc. A-Math. Phy. 274 (1963) 21.

[2] T. Nakahigashi, Y. Tanaka, K. Miyake, H. Oohara, Tribol. Int. 37 (2004) 907.

[3] Y.T. Pei, X.L. Bui, X.B. Zhou, J.Th.M. De Hosson, J. Vac. Sci. Technol. A 26 (2008) 1085.

[4] Y. Aoki, N. Ohtake, Tribol. Int. 37 (2004) 941.

[5] Y.T. Pei, X.L. Bui, J.P. van der Pal, D. Martinez-Martinez, J.Th.M. De Hosson, Acta Mater. 60 (2012) 5526.

[6] B.N. Persson, J. Phys. - Condens. Matter 18 (2006) 7789.

[7] F.P. Bowden, D. Tabor, The friction of and lubrication of solids. Clarendon Press, Oxford, 1964.

[8] D.F. Moore, The friction and lubrication of elastomers. Pergamon Press, 1972. 
156 Surface Effects and Contact Mechanics XI

[9] D. Martinez-Martinez, J.P. van der Pal, Y.T. Pei, J.Th.M. De Hosson, J. Appl. Phys. 110 (2011) 124907.

[10] K.L. Johnson, Contact Mechanics. Cambridge University Press, Cambridge, 1985.

[11] W.D. May, E.L. Morris, D. Atack, J. Appl. Phys. 30 (1959) 1713. 